\title{
Cloning and Expression of cry9Ea10 Gene from Bacillus Thuringiensis Strain GZ2 Isolated from Infected Hyphantria Cunea Larvae
}

\author{
Kun-li ZHAO ${ }^{1}$, Wei GUO ${ }^{1,2, *}$, Dan ZHAO $^{1}$,Ya-kun ZHANG ${ }^{1}$, \\ Xiao-ping YAN $^{1}$ and $Y u$-jie $\mathrm{GAO}^{1}$ \\ ${ }^{1}$ College of Plant Protection, Agricultural University of Hebei, Baoding 071001, China \\ ${ }^{2}$ Plant Science and Technology College, Beijing University of Agriculture, Beijing \\ 102206, China
}

Keywords: Bacillus thuringiensis, Cry gene,Infected Hyphantria cunea, Biological control.

\begin{abstract}
A novelBacillus thuringiensis(Bt) strain GZ2, was isolated from infected Hyphantria cunealarvae. The bioassay results showed that GZ2 had insecticidal activity to H.cunea larvae. The genotypes ofGZ2 were identified by PCR-RFLP method. GZ2 was identified to harbor cry9Ealo gene and the full length of cry9EalO was cloned and sequenced. The engineering strain of Bt carrying cry9Ealogene wasconstructed successfully. SDS-PAGE analysis confirmed that the expression of cry9Ea10 as $\sim 130 \mathrm{kDa}$ protein. And Cry9Ea10 protein produced by $\mathrm{Bt}$ engineeringstrainexhibited a high toxicity to H.cunealarvae.
\end{abstract}

\section{Introduction}

Hyphantria cunea Drury was native to North America then spread to Europe and Asia due to human activities [1]. This international quarantine pest has caused tremendous damages to forest in China since 1979 because of its high reproductivecapability, good adaptability and wide reach [2,3]. The chemical control against H.cunea are deleterious to the environment and the health of humans [4,5]. So alternative methods for pest control that are less hazardous are highly valued.

Bacillus thuringiensis(Bt)bacteria are one of the best substitutes, which produceinsecticidal crystalline proteins(ICPs) during the sporulation phase of growth. Different ICPs are highly specific to target insects [6]. After larvae ingestion, the crystalline inclusions are solubilized in midgut lumen and activated by alkalineproteases releasing $65-\mathrm{kDa}$ toxic fragment that binds to severalmidgut receptors. The interactions facilitate toxin insertion into the membrane, forming pores that result in the death of mid gut cells $[7,8]$.

Soil is the most common source to sift Bt strainson account of carrying with vast microorganism. However, it's a long period to get a strainthat has specific and high toxicity to a target pest. In the present study, We put attention to the naturally infected $H$. cunea larvae collected from the wild which show classic symptom of infection by bacteria. A novel cry9Eagenewas cloned and expressed from one of the isolates detected from infected $H$. cunea larvae, the crystal protein purified from engineering Bt strain have high toxicity against $H$. cunea larvae.

\section{Materials and Methods}

\section{Hyphantria Cunea Larvae}

The infectedlarvae were collected from Platanus orientalis wood out of pesticides in 
Zhengding(Hebei, China) and fed by Ailanthus altissima leaves in lab. The healthy ones were provided by Chinese Academy of Forestry and fed by artificial diet.

\section{Isolation and Identifica tion of Bt strains}

After 15 minutes surface sterilization by $75 \%$ alcohol, the infected $H$. cunea larvae were put in the Milli-Q water and vortexed to get the tissue homogenate. Shake for 20 minutes at room temperature (at $200 \mathrm{rpm}$ ). Then incubate in $75^{\circ} \mathrm{C}$ water bath for 17 minutes. After cold to room temperature, dilute the supernate to $10^{-2}, 10^{-3}, 10^{-4}$ three gradients and pipette $100 \mu 1$ to spread on $1 / 2$ LB solid medium separately. Incubate at $30^{\circ} \mathrm{C}$ for three days and pick up the white irregular colony with waxy rough surface. The microorganism wasidentified under microscope after carbolfuchsin staining.

The colonies were cultivated in 1/2 LB liquid medium for 72 hours. After centrifugation, the supernate was removed and the sediment was resuspended in $10 \mathrm{mM}$ Tris- $\mathrm{HCl}$ ( $\mathrm{pH} 8.0)$. The samples were added protein sample buffers boiling for 10 minutes. Proteins produced by the wild Bt strains were analyzed by SDS-PAGE.

\section{Identification of cry Gene Types}

27 pairs of universal oligonucleotide primers were used to detect the gene types by PCR [9]. The amplified PCR fragments were purified using Universal DNA Purification Kit (Tiangen, China) and the restriction fragment length polymorphism (RFLP) patterns were performed.

\section{Cloning of the Full Length of cry9Ea10}

Specific primers were then designed depending on the blast in NCBI. The full length gene was cloned and sequenced. The result was then submitted to Bacillus thuringiensis Toxin Nomenclature Committee and NCBI to acquire a name and GenBank accession number respectively.

\section{Expression of Cry9Ea10 in Escherichia coli}

The purified fragments of cry9Eal0 with restrict enzyme sites were cloned into pET21b vector [10]. The recombined plasmid was transformed into E. coli BL21(DE3) competent cells. Protein expression was induced by the addition of 1 $\mathrm{mM}$ IPTG for 6hours. After centrifugation, the precipitates were resuspended with $10 \mathrm{mM}$ Tris-HClbuffer $(\mathrm{pH}=8.0)$ and sonicated to release proteins.

The expressed proteins were separated by $8 \%$ SDS-PAGE and transferred to PVDFmembrane. The PVDF membrane were blocked for $1 \mathrm{~h}$ with $1 \%$ BSA, incubated with mouse anti-His (1:2000)for $1 \mathrm{~h}$, washed four times with TBS-T and two times with TBS. The protein was detected using anti-mouse conjugated with Phosphatase alkaline(1:10000). Finally, NBT and BCIP (Sigma) was used as substrate for detection.

\section{Expression and Purification of Cry9Ea10 in HD73-}

The full-length fragments of cry9EalO with the restrict enzyme sites were cloned into pSXY422b vector. The recombined plasmid were transformed into acrystalliferous mutant HD73- competent cells by electric shock at $2500 \mathrm{~V}, 5.0 \mathrm{~ms}$.

The expressed Cry9Ea10 protein after culturing for 72 hours in $1 / 2 \mathrm{LB}$ was purified with isoelectric precipitation. The concentration of the purified Cry9Ea10 was measured by BCA method according to the manufacturer's instructions (TAKARA).

The purified Cry9Ea10 was analyzed by Western Blot (as in the last section). The 
membrane was revealed using rabbit anti-cry9 $\mathrm{Ea}(1: 20000)$ and secondary anti-rabbit conjugated with Phosphatase alkaline (1:10000).

\section{Insect Bioassays}

Bioassays were performed with $H$. cunea larvae by the diet surface contamination method [6]. 20 primary hatchinglarvae/box were fed with different doses of GZ2 spores or Cry9Ea10 proteins and 3 repeats were conducted. The boxes were incubated at $28^{\circ} \mathrm{C}$ with $65 \pm 5 \%$ relative humidity. Mortality was recorded after 96 hours and the $50 \%$ lethal concentration $\left(\mathrm{LC}_{50}\right)$ was analyzed with SPSS software.

\section{Results}

\section{Isolation and Identification of $\mathrm{Bt}$}

Two Bt isolates were gained. SDS-PAGE shows they both produce about $130 \mathrm{kDa}$ bands(Fig. 1, A and B).

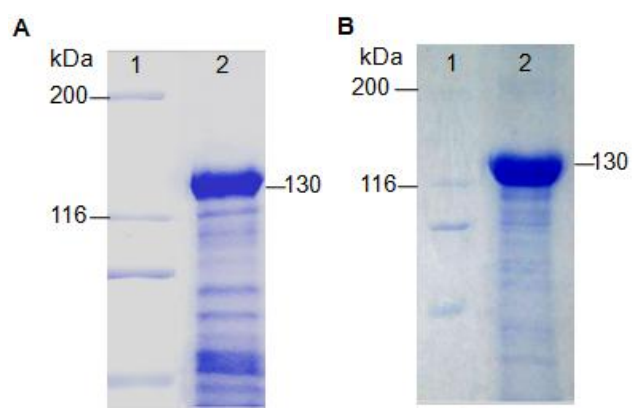

Fig. 1 SDS-PA GE analys is of proteins produced by wild Bt isolates. A, isolate GZ1(lane 2), Lane 1, molecular weight marker. B, isolate GZ2(lane 2), Lane 1, molecu lar weight marker.

\section{Cloning and Analysis of cry9Ea10 Gene}

The conservative regions of several cry genes were obtained by universalprimersand the full-length sequence of $\operatorname{cry} 9 E$ Eal0was cloned successfully by specific primers. The gene was named as cry9EalOby Bacillus thuringiensis Toxin Nomenclature Committee. The GenBank accession number is KT692743.

The ORFof cry9Eal0 is $3453 \mathrm{bp}$, encoding 1150 amino acids, with a calculated molecular mass of $130.0 \mathrm{kDa}$, and isoelectric point $(\mathrm{pI})$ of 4.68 . Amino acid sequence analysis using the NCBI ConservedDomain Database, showed that the Cry9Ea10 protein includes three putative conserved Domains(Fig. 2A). The 3D-modeled structures of Cry9Ea10 were predicted by SWISS-MODLE on line (Fig. 2B).

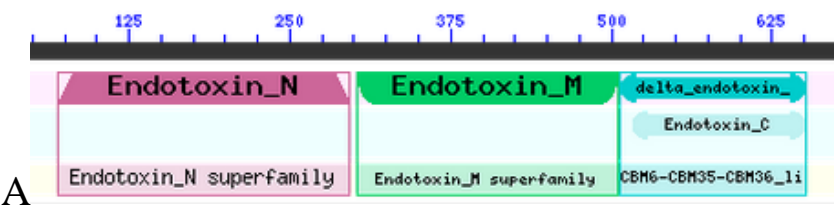

B

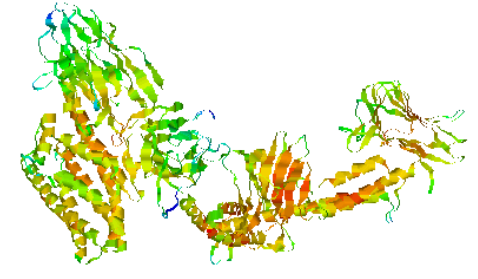

Fig. 2 The binary and ternary structure of Cry 9Ea10. A, three putativeDomains of Cry 9Ea 10. B, 3D-modeled structures of Cry9Ea 10 .

\section{Expression of pET21b-9Ea10 in BL21}

The pET21b-9Ea10 was constructed and transferred into BL21successfully (Fig. 3A). Western Blot analysis showed that the molecular weight of expressed Cry 9 Ea10 protein was about $130 \mathrm{kDa}$ (Fig. 3B), which was coherent with the predicted 
molecular weight.
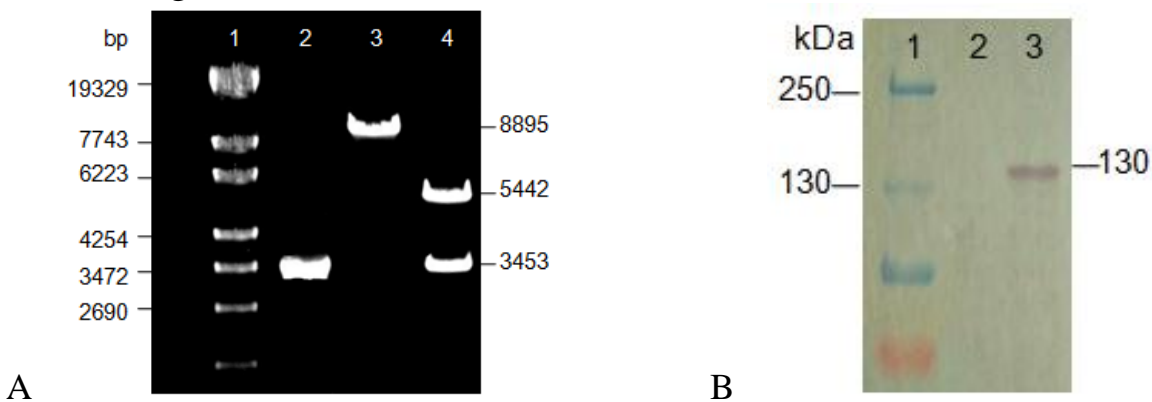

Fig. 3 The pET21b-9Ea10was successfully constructed and expressed in BL21. A, identification of the recomb inant plasmid. Lane 1, marker. Lane 2, PCR product. Lane 3, single endonuclease digestion. Lane4, double endonuclease digestion.B, Western Blot analysis of the recombinant protein. Lane 1, prestained marker.Lane 2, the expression of pET21b. Lane 3, the expression of pET21b-9Ea 10.

\section{Expression and Purification of Cry9Ea10 in HD73-}

The pSXY422b-9Eal0 was successfully constructed (Fig. 4A). The heterogenous expressed protein Cry9Ea10 was purified and Western Blot analysis showed a $130 \mathrm{kDa}$ band (Fig. 4, B and C). The concentration of the purified Cry9Ea10 was measured as $3.5 \mathrm{mg} / \mathrm{ml}$ by BCA method.
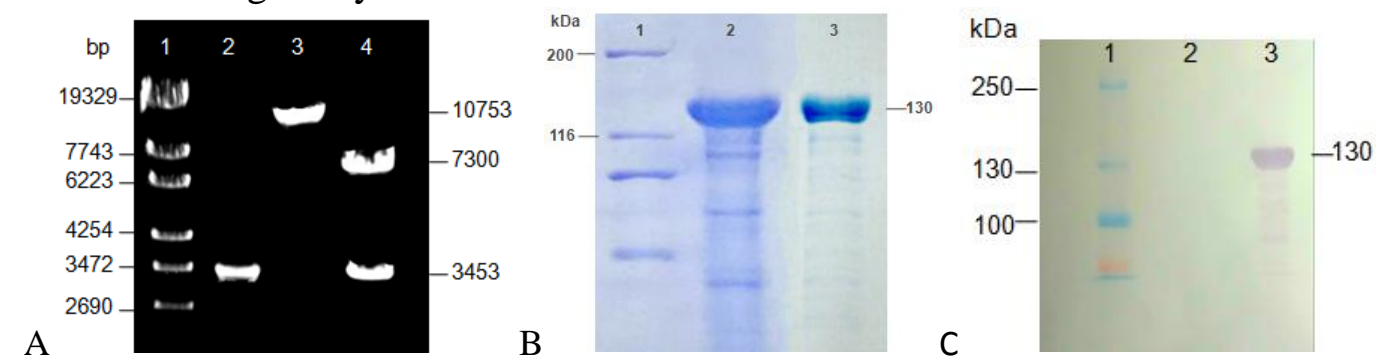

Fig. 4 The pSXY422b-9Ea10was successfully constructed and expressed in HD73-. A, identification of the recombinant plas mid. Lane 1, marker.Lane 2, PCR product. Lane 3, single endonuclease digestion.

Lane 4, double endonuclease digestion.B, coomassie blue staining of the heterogenous expressed protein Cry 9Ea 10.Lane 1, molecular weight marker. Lane 2, the exp ression of HD73

(pSXY422b-9Ea10). Lane 3, the purified protein Cry9Ea 10. C, Western Blot analysis of the purified protein Cry 9Ea 10. Lane 1, prestained marker. Lane 2, the expression of HD73. Lane 3, the purified protein Cry $9 \mathrm{Ea} 10$.

\section{Insect Bioassays}

The mortality of GZ2 strain and Cry9Ea10 protein to H.cunea was calculated and analyzed respectively (Fig. 5). The $\mathrm{LC}_{50}$ lethal values of isolate GZ2 were 1921 $\mathrm{pfu} / \mathrm{ml}$. The $\mathrm{LC}_{50}$ lethal values of Cry9Ea10 proteinwere $4.14 \mu \mathrm{g} / \mathrm{ml}$. 

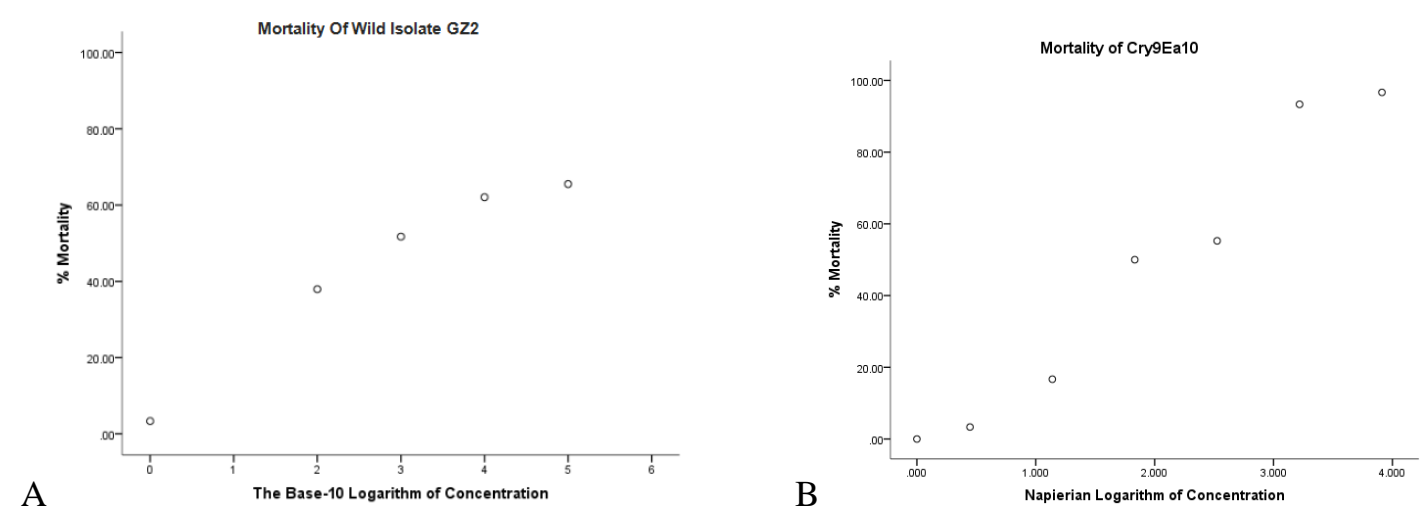

Fig. 5 Toxicity effects to $H$. cunea larvae. A, bioassay of Bt isolate GZ2 to H. cunea larvae.Horizontal ordinate, the base-10 logarithm of concentration. Vertical coordinates, mortality. B, bioassay of Cry9Ea10 proteinto H.cunea larvae. Horizontal ordinate, the natural logarithm of concentration. Vertical coordinates, mortality.

\section{Discussion}

In this study, a novelBt strain isolated from the naturally infected $H$. cunea does have high toxicity to healthy $H$. cunealarvae. It provides an effective insecticidal crystal protein to control $H$. cuneaand study the interactions between Cry proteins and receptors on BBMV of insect midgut.

Previous research had indicated that Cry9Ea is highly toxic to Trichoplusia $n i$ neonates $\left(\mathrm{LC}_{50}=0.0639 \mu \mathrm{g} / \mathrm{mL}\right)$ and Plutella xylostella neonates $\left(\mathrm{LC}_{50}=4.574 \mu \mathrm{g} / \mathrm{mL}\right)$ [11], but not to Helicoverpa armigera. According to the Ping Pong binding model, the activated cry monomer first binds to APN[12]. The 3D structures of APNfrom $T$. ni, H. cunea, P. xylostellaand $H$. armigerapredicted by SWISS MODELwere compared. The results showed thatthere is a deep crack between Domain Gluzincin and Domain ERAP1_C in T. ni APN. In H. cuneaand P. xylostellaAPN, Domain ERAP1_C inserts into Domain Gluzincin further and two Domains joint more closely, which may block the bind between Cry9Ea and APN to some extent. In H. armigera, two Domains are bounded tightly together and the crack is totally blocked. These differencemay indicate how Cry9Ea bind to APN on the configuration.

However, the intermolecular mechanism between Cry9Ea and several receptors is still remained to be understood. The future research will help to understand the key site of interactions and obtain insecticides of higher toxicity, which would be of great value for the biological control of $H$. cunea.

\section{Reference}

[1] Umeya K, I.Y. et al. Adaptation and Speciation in the Fall Webworm: Invasion and establishment of a new insect pest in Japan. Kodansha. 1-12 (1977).

[2] Rong J, X. et al. The progress research of exotic species Hyphantria cunea. Entomological Science. 40, 13-18 (2003).

[3] Sharifi, M.et al. Effects of 4-hexylresorcinol on the phenoloxidase from Hyphantria cunea. Insect Science. 22, 639-650 (2015).

[4] Talebi, K.H, V. et al. Ecological impacts of the pesticides in agricultural ecosystem. Pesticides in Modern Worlds. 143-168 (2011). 
[5] Devine GJ, F.M.et al. Insecticide use: contexts and ecological consequences. Agric Human Values. 24, 281-306 (2007).

[6] Bravo A, G. et al. Oligomerization triggers binding of a Bacillus thuringiensisCry1 $\mathrm{Ab}$ pore-forming toxin to aminopeptidase $\mathrm{N}$ receptor leading to insertion into membrane microdomains. BBA. 1667, 38-46 (2004).

[7] Pardo-Lopez, L. et al.Bacillus thuringiensisinsecticidal three-domain Cry toxins. Fems Microbiol Rev. 37, 3-22 (2013).

[8] Gomez, I. et al. Cadherin- like receptor binding facilitates proteolytic cleavage of helix alpha-1 in domain I and oligomer pre-pore formation of Bacillus thuringiensisCry1 Ab toxin. Febs Letters. 513, 242-246 (2002).

[9] Kuo, WS. et al. Identification of novel cry-type genes from Bacillus thuringiensis strains on the basis of restriction fragment length polymorphism of the PCR-amplified DNA. Appl Environ Microbiol. 62, 1369-1377 (1996).

[10]Jimenez-Juarez, N. et al. Bacillus thuringiensis Cryl Ab mutants affecting oligomer formation are non-toxic to Manduca sexta larvae. J Biol Chem. 282, 21222-21229 (2007).

[11] Tinghui, L. et al. Study on Bacillus thuringiensis strain Bt11 and GS8 Applicant (In Chinese).

[12]Pacheco, S. et al. Domain II Loop 3 of Bacillus thuringiensis Cry1 Ab Toxin Is Involved in a "Ping Pong" Binding Mechanism with Manduca sexta Aminopeptidase-N and Cadherin Receptors. J Biol Chem. 284, 32750-32757 (2009). 